\title{
Perinatal Management of Bart's Hemoglobinopathy: Paradoxical Effects of Intrauterine, Transplacental, and Partial Exchange Transfusions
}

\author{
Mark Curran, MD ${ }^{1}$ Michel Mikhael, MD ${ }^{2,3}$ Wang-Dar Sun, MD ${ }^{2,3}$ Jina Lim, MD ${ }^{2,3}$ Anna Leung, MD ${ }^{1}$ \\ Gira Morchi, MD ${ }^{4}$ Ramen H. Chmait, MD
}

${ }^{1}$ Division of Maternal Fetal Medicine, Pomona Valley Hospital Medical Center, Pomona, California

${ }^{2}$ Division of Neonatology, Pomona Valley Hospital Medical Center, Pomona, California

${ }^{3}$ Neonatal-Perinatal Medicine Division, Children's Hospital of Orange County, Orange, California

${ }^{4}$ Division of Pediatric Cardiology, Children's Hospital of Orange County, Orange, California

${ }^{5}$ Division of Maternal-Fetal Medicine, Department of Obstetrics and Gynecology, Keck School of Medicine, University of Southern California, Los Angeles, California

Address for correspondence Wang-Dar Sun, MD, Neonatal-Perinatal Medicine Division, Children's Hospital of Orange County, 1201 W La Veta Ave, Orange, CA 92868 (e-mail: WSun@choc.org).

Am J Perinatol Rep 2020;10:e11-e14.

\begin{abstract}
Keywords

- hemoglobin Bart

- fetal transfusion

- partial exchange transfusion

We describe a fetus at $24{ }^{3 / 7}$ weeks' gestation that showed ultrasound evidence of anemia, hydrops, and severe growth restriction. Both parents were known to be cis heterozygous carriers for SEA $\alpha$-thalassemia deletion $(\alpha \alpha /-)$. Cordocentesis confirmed fetal anemia and homozygous $\alpha$-thalassemia $(-1-)$ in the fetus. Fetal intrauterine transfusions corrected the anemia, treated the hydrops, and improved fetal growth. The postnatal course was complicated by hypoxic respiratory failure and persistent pulmonary hypertension of the newborn, which resolved only after partial volume exchange transfusion. This case report is presented to point out the potential unintended outcomes with transplacental transfusion via delayed cord clamping and cord milking at delivery in the setting of congenital Bart's hemoglobinopathy, and demonstrates that partial exchange transfusion of the newborn may optimize oxygen delivery due to the more favorable oxygen affinity of transfused adult hemoglobin compared with the Bart's hemoglobin.
\end{abstract}

Defective or absent production of all $\alpha$-globin chains, as occurs in $\alpha$-thalassemia major, leads to the formation of gamma-globin tetramers called hemoglobin Bart (Hb Bart) in the fetus. Hb Bart has an extremely high oxygen affinity and cannot deliver oxygen to the fetus effectively. Fetuses affected by Bart's hemoglobinopathy develop generalized edema, pleural, and pericardial effusions (hydrops fetalis) due to severe hypoxia, and usually die in utero or shortly after birth. In addition, mothers carrying a fetus with hydrops fetalis are at increased risk of hypertension, pre- eclampsia, antepartum hemorrhage, renal failure, premature labor, and abruptio placenta. ${ }^{1}$ Timely prenatal diagnosis and counseling are crucial to helping families decide upon high risk but potentially lifesaving fetal intervention. Even with increased survival due to intrauterine transfusions (IUT), ${ }^{2}$ there is a lack of understanding of physiologic and developmental alterations related to the disease, as well as poorly described postnatal practices. We detail our experience in this case to share new insights into prenatal and postnatal management of $\mathrm{Hb}$ Bart's hydrops fetalis. received

April 10, 2019

accepted after revision

October 8, 2019
DOI https://doi.org/

10.1055/s-0039-3401799. ISSN 2157-6998.
Copyright $\odot 2020$ by Thieme Medical Publishers, Inc., 333 Seventh Avenue, New York, NY 10001, USA. Tel: +1(212) 760-0888.
License terms

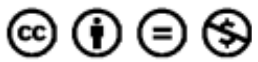


e12 Hemoglobin Bart and Neonatal Respiratory Failure Curran et al.

Table 1 Hemoglobin level obtained over time

\begin{tabular}{|l|l|l|l|l|}
\hline & $\begin{array}{l}\text { GA } \\
(\mathbf{w k})\end{array}$ & $\begin{array}{l}\text { Pretransfusion } \\
\text { hemoglobin } \\
(\mathbf{g} / \mathbf{d L})\end{array}$ & $\begin{array}{l}\text { Posttransfusion } \\
\text { hemoglobin } \\
(\mathbf{g} / \mathbf{d L})\end{array}$ & $\begin{array}{l}\text { Transfusion } \\
\text { volume } \\
(\mathbf{m L})\end{array}$ \\
\hline IUT 1 & $25^{2 / 7}$ & 7.9 & 11.1 & 25 \\
\hline IUT 2 & $26^{2 / 7}$ & 11.7 & 13.7 & 20 \\
\hline IUT 3 & $28^{2 / 7}$ & 10.6 & 13.6 & 36 \\
\hline IUT 4 & $31^{2 / 7}$ & 11.0 & 14.1 & 50 \\
\hline IUT 5 & $34^{2 / 7}$ & 11.3 & 14.4 & 60 \\
\hline DOL1 & 37 & 19 & $\mathrm{NA}$ & $\mathrm{NA}$ \\
\hline
\end{tabular}

Abbreviations: DOL, day of life; GA, gestational age; IUT, intrauterine transfusion; NA, not applicable.

\section{Case Report}

A 27-year-old Filipino woman, gravida 1 , para 0 at $20^{1 / 7}$ weeks' gestation was referred to perinatology due to maternal SEA heterozygote deletion $(\alpha \alpha /-)$ mutation. Additional testing showed her partner was also a cis heterozygous carrier for the SEA $\alpha$-thalassemia deletion $(\alpha \alpha /-)$. The parents were advised of the $25 \%$ risk to their fetus of having $\alpha$-thalassemia major. They declined amniocentesis and opted to have expectant management. Ultrasound of the fetus on her initial visit was unremarkable.

A follow-up ultrasound of the fetus at $24^{3 / 7}$ weeks' sonogram showed oligohydramnios, shortened long bones, and severe cardiomegaly with a cardiothoracic circumference ratio of 0.65 . Doppler velocimetry showed normal forward flow in the umbilical artery, and a middle cerebral artery peak systolic velocity of 1.49 multiples of the median. The patient was referred to a tertiary center for suspected Bart's hemoglobinopathy.

Examination at the referral center found the fetus to have moderate hydrops in addition to the previous findings. The patient was informed that the most likely diagnosis was ATM. After discussion of the risks of IUT, the need for chronic postnatal transfusion, possible bone marrow transplantation, and increase risk for neurodevelopmental impairment, the parents elected to proceed with serial IUTs. On initial cordocentesis, the fetal hemoglobin level measured $7.9 \mathrm{~g} / \mathrm{dL}$. Fetal electrophoresis showed 35.3\% Hb A1 and 62.5\% Hb Bart. IUT was performed and the follow-up ultrasound at $27^{1 / 7}$ weeks showed resolving hydrops and symmetric fetal growth restric- tion with estimated fetal weight at the 2.7 th percentile. By 30 $1 / 7$ weeks, hydrops and oligohydramnios had resolved and the fetal weight was at the 29th percentile. A total of five IUTs were performed over the course of the pregnancy. The fetal hemoglobin levels and hemoglobin electrophoresis results are shown in - Tables 1 and 2, respectively.

At 37 weeks' gestation, the mother was induced and delivered vaginally a live, well appearing, 2,984 g, female infant. Cord milking was performed after delivery per standard practice. Delivery room care included blow by oxygen and face mask continuous positive pressure (CPAP) for cyanosis. Upon admission to the neonatal intensive care unit, the infant was placed on nasal CPAP for continued oxygen requirement. Empiric ampicillin and gentamicin were started. Initial complete blood count was significant for $\mathrm{Hb}$ of $19 \mathrm{~g} / \mathrm{dL}$ and hematocrit of $64 \%$.

Within the first hour of life, the neonate continued to be hypoxemic and required as high as 0.7 fraction inspired oxygen $\left(\mathrm{FiO}_{2}\right)$. She had normal work of breathing and no signs of respiratory distress. Chest X-ray (CXR) showed mild ground glass opacities with adequate lung expansion. Arterial blood gas was unremarkable with $\mathrm{pH} 7.38, \mathrm{PCO}_{2} 38 \mathrm{~mm} \mathrm{Hg}$, and $\mathrm{PO}_{2}$ $95 \mathrm{~mm} \mathrm{Hg}$ on nasal CPAP with $\mathrm{FiO}_{2}$ at 0.67 . Pulse oximetry in the lower extremities was 5 to $10 \%$ lower than the upper extremities. Oxygenation index was calculated $\left(\mathrm{FiO}_{2} \times\right.$ mean air way pressure/arterial oxygen partial pressure) to be 3.5 , and alveolar-arterial oxygen gradient was $357 \mathrm{~mm} \mathrm{Hg}$. Echocardiogram showed evidence of elevated pulmonary vascular resistance with estimated right ventricular systolic pressure of $65 \mathrm{~mm} \mathrm{Hg}$, bidirectional shunts through the patent ductus arteriosus and patent foramen ovale, flattened interventricular septum, and no left heart obstructive lesion or myocardial dysfunction. Inhaled prostacyclin (PGI2) was started during the echocardiogram, with an immediate response demonstrated by a $10 \mathrm{~mm} \mathrm{Hg}$ decrease in estimated right ventricle pressure.

With inhaled PGI2 treatment via nasal CPAP, the patient's oxygen requirement decreased within 1 hour to $0.48 \mathrm{FiO}_{2}$, and was weaned to 0.25 over the next 24 hours. Inhaled PGI2 was weaned off at 24 hours of life with sustained improvement in oxygenation. The patient was placed on high-flow nasal cannula on day of life (DOL) 2, then weaned to room air on DOL 5. However, DOL 6 had desaturations into the 80s necessitating oxygen therapy by low-flow nasal cannula. Over the next 14 days, infant continued to require oxygen between 0.21 and $0.3 \mathrm{FiO}_{2}$. During this time, echocardiogram (DOL 12) demonstrated normalization of RV pressures.

Table 2 Hemoglobin electrophoresis obtained prior to fetal transfusions and at birth

\begin{tabular}{|l|l|l|l|l|l|l|}
\hline & GA (wk) & Hemoglobin A (\%) & Hemoglobin A2 (\%) & Hemoglobin F (\%) & $\begin{array}{l}\text { Hemoglobin } \\
\text { Bart (\%) }\end{array}$ & Other hemoglobin (\%) \\
\hline IUT 1 & $25^{2 / 7}$ & 0.0 & 0.0 & 0.0 & 96.8 & Portland 3.2 \\
\hline IUT 2 & $26^{2 / 7}$ & 35.2 & 0.9 & 0.0 & 62.6 & H 1.2 \\
\hline IUT 3 & $28^{2 / 7}$ & 67.6 & $<1.0$ & 0.0 & 30.8 & Other 1.6 \\
\hline IUT 4 & $31^{2 / 7}$ & 49.1 & 1.3 & 0.0 & 47.0 & H 2.6 \\
\hline DOL1 & 37 & 50.0 & 1.3 & 3.0 & 45.0 & 0.0 \\
\hline
\end{tabular}

Abbreviations: DOL, day of life; GA, gestational age; IUT, intrauterine transfusion. 
The primary pediatric hematologist was consulted. Though the plan established prenatally was to transfuse within a week after delivery, the patient's high hemoglobin (ranging from $19 \mathrm{~g} / \mathrm{dL}$ and slowly decreasing to $15 \mathrm{~g} / \mathrm{dL}$ ) precluded this. Hemoglobin electrophoresis from DOL 1 resulted as $45 \% \mathrm{Hb}$ Bart, $50 \% \mathrm{Hb} \mathrm{A}, 1.3 \% \mathrm{Hb} \mathrm{A} 2$, and 3\% $\mathrm{Hb} \mathrm{F}$. It was felt that the high $\mathrm{Hb}$ Bart's percentage was contributing to the infant's oxygen requirement by reducing oxygen delivery. On DOL 18 , it was decided to perform a partial volume exchange of $20 \mathrm{~mL} / \mathrm{kg}$ with normal saline to bring down hemoglobin level, and then transfuse packed red blood cells. Prior to the volume exchange, the $\mathrm{Hb}$ was $14.7 \mathrm{~g} / \mathrm{dL}$. After the partial volume exchange and red blood cell transfusion, the $\mathrm{Hb}$ remained stable at $14.8 \mathrm{~g} / \mathrm{dL}$. One day after the red blood cell transfusion, the patient's oxygenation improved and the baby was weaned to room air.

Other aspects of the newborn's care included a sepsis evaluation at birth and initiation of empiric ampicillin and gentamicin. Antibiotics were stopped with negative cultures at 48 hours. On DOL 2, bilirubin level reached a peak of $13.6 \mathrm{mg} / \mathrm{dL}$ for which she received phototherapy for 24 hours. The infant was discharged 2 days after weaning to room air with close follow-up with pediatric hematology within 2 weeks to set up future transfusions.

At 16 months of age, the patient has exhibited appropriate somatic growth for age and normal developmental milestones. She continues to receive chronic blood transfusions every 3 weeks. Her only other known medical problem is eczema. Her only chronic medication is daily multivitamin. She is currently awaiting bone marrow transplantation.

\section{Discussion}

Despite improvement in fetal growth and resolution of hydrops after serial IUT, our patient had significant short term postnatal complications requiring neonatal intensive care unit intervention for hypoxic respiratory failure and persistent pulmonary hypertension of the newborn (PPHN). This infant's postnatal course, therefore, merits discussion, as there is little literature to describe optimal postnatal management in this patient population.

Delayed cord clamping (DCC) or cord milking immediately after delivery has not been studied in this patient population. Due to known and well-studied benefits for term and preterm infants, this practice has become standard of care in all healthy infants as per American College of Obstetricians and Gynecologists 2017 recommendations. ${ }^{3}$ However, the benefit is less studied in fetuses with hematologic pathology resulting in anemia. In a small study by Garabedian et al looking at 72 fetuses with anemia due to red cell alloimmunization requiring IUT, short-term benefits of DCC were found. ${ }^{4}$ These include fewer postnatal exchange transfusions and higher hemoglobin level, without any differences in severity of hyperbilirubinemia.

In our patient, cord milking was performed to facilitate resuscitation. The resultant hemoglobin level at birth was high at $19 \mathrm{~g} / \mathrm{dL}$. Interestingly, despite five IUT, the relative percentage of $\mathrm{Hb}$ Bart remained relatively high ( $45 \% \mathrm{Hb}$ Bart). The presence of polycythemia deterred the clinical team from performing an early transfusion of packed red blood cells (PRBCs). However, in retrospect, the relatively high $\mathrm{Hb}$ Bart concentration resulted in tissue hypoxia and the secondary respiratory complications of hypoxic respiratory failure and PPHN. Only after undergoing a partial volume exchange transfusion on DOL 18 did tissue oxygenation improve, resulting in a quick recovery of the neonate.

In contrast to patients with alloimmune anemia whose hemoglobin is fully functional in oxygen delivery, patients with $\mathrm{Hb}$ Bart have a proportion of hemoglobin which cannot effectively deliver oxygen. Therefore, more endogenous hemoglobin may not be beneficial in the physiology of Hb Bart. While we cannot state, based on this one case, that DCC or cord milking is contraindicated in the setting of Bart's hemoglobinopathy, our experience leads us to believe that more studies should be performed to evaluate its benefit in this particular setting. Until such studies are done, the decision on DCC should be individualized and discussed between the obstetrician and neonatologist.

This infant's postnatal course may be related to alterations in fetal physiology and should be discussed. In this case IUT was lifesaving to the fetus and reversed growth restriction and hydrops. However, even after multiple transfusions, $\mathrm{Hb}$ A1 accounted for only about half of the fetus's hemoglobin, with the majority of the remainder comprised of Hg Bart. Prolonged and significant presence of Bart's hemoglobin in the fetal circulation can potentially result in chronic fetal hypoxemia due to altered oxygen delivery characteristics. Fetal hypoxemia has been demonstrated to cause pulmonary vasoconstriction and increase pulmonary vascular resistance. ${ }^{5}$ In fact, vascular beds of multiple organ systems can be affected. Fetuses with prolonged in utero hypoxemia exhibit cardiovascular adaptations that redistribute blood flow to the brain, myocardium, and adrenal glands. ${ }^{6}$ This phenomenon may begin to explain the high risk of PPHN as well as adverse long-term growth and developmental outcomes even after the benefit of IUT. ${ }^{7}$ In spite of improvement in fetal status after IUT, these newborns may have immediate and acute need for resuscitation, supplemental oxygen, mechanical ventilation, and pulmonary vasodilator therapy.

In summary, we presented an infant with severe Bart's hemoglobinopathy who received several IUTs due to anemia and hydrops. Her hydrops was resolved, but her postnatal course was complicated by respiratory failure and pulmonary hypertension possibly due to alteration of fetal physiology and oxygen delivery. Her condition was improved only after partial exchange transfusion. Thus, prenatal and postnatal management of $\mathrm{Hb}$ Bart requires both resolution of anemia and reduction of the relative percentage of Bart's hemoglobin, as both measures serve to increase oxygen delivery. Better understanding of how the disease affects fetal physiology, as well as studies on postnatal management, are needed to refine perinatal and neonatal care for this growing population and ultimately improve long-term outcomes.

\section{Statement of Ethics}

Parents have given written informed consent to publish this case report regarding their child. 
e14 Hemoglobin Bart and Neonatal Respiratory Failure Curran et al.

\section{Contributors}

All authors have made substantial contributions to the conception and design of the manuscript, as well as the writing and approval of the final version of the manuscript.

\section{Funding}

None.

\section{Conflict of Interest}

None declared.

\section{References}

1 Vichinsky EP. Alpha thalassemia major-new mutations, intrauterine management, and outcomes. Hematology Am Soc Hematol Educ Program 2009:35-41. Doi: 10.1182/asheducation-2009.1.35
2 Chmait RH, Baskin JL, Carson S, Randolph LM, Hamilton A. Treatment of alpha(0)-thalassemia (-(SEA)/-(SEA)) via serial fetal and post-natal transfusions: can early fetal intervention improve outcomes? Hematology 2015;20(04):217-222

3 Committee on Obstetric Practice. Committee opinion no. 684: delayed umbilical cord clamping after birth. Obstet Gynecol 2017; 129(01):e5-e10

4 Garabedian C, Rakza T, Drumez E, et al. Benefits of delayed cord clamping in red blood cell alloimmunization. Pediatrics 2016;137 (03):e20153236

5 Vali P, Lakshminrusimha S. The fetus can teach us: oxygen and the pulmonary vasculature. Children (Basel) 2017;4(08):E67

6 Maršál K. Physiological adaptation of the growth-restricted fetus. Best Pract Res Clin Obstet Gynaecol 2018;49:37-52

7 Songdej D, Babbs C, Higgs DR; BHFS International Consortium. An international registry of survivors with Hb Bart's hydrops fetalis syndrome. Blood 2017;129(10):1251-1259 